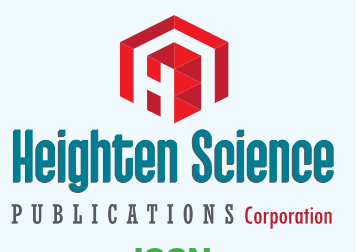

ISSN

2573-7724
Research Article

\section{Clinically and Radiological isolated syndrome (MS risk)}

\author{
Hassan Ahmed Hashem ${ }^{1 *}$, Yasser Hamed Mustafa ${ }^{1}$, \\ Abdelazim M Reda ${ }^{2}$, Sameh Azab ${ }^{3}$ and Ahmed M Solaiman ${ }^{4}$ \\ ${ }^{1} \mathrm{MD}$, Lecturer of Neurology, Al Azhar Faculty of Medicine, Assiut, Egypt \\ ${ }^{2} \mathrm{MD}$, Lecturer of Neurology, Faculty of Medicine, Cairo University, Egypt \\ ${ }^{3} \mathrm{MD}$, Lecturer of Radiology, Faculty of Medicine, El-Menofia University, Egypt \\ ${ }^{4} \mathrm{MD}$, Lecturer of Clinical Pathology, Al Azhar Faculty of Medicine, Assiut, Egypt
}

*Address for Correspondence: Hassan Ahmed Hashem, MD, Lecturer of Neurology, Al Azhar Faculty of Medicine, Assiut, Egypt, Tel: 00966540691003; Email: hassol1975@yahoo.com

Submitted: 10 July 2018

Approved: 27 July 2018

Published: 28 July 2018

Copyright: @ 2018 Hashem HA, et al. This is an open access article distributed under the Creative Commons Attribution License, which permits unrestricted use, distribution, and reproduction in any medium, provided the original work is properly cited.

Keywords: RIS; CIS; MS; MRI; CSF analysis

\section{Abstract}

Background: The use of brain magnetic resonance imaging (MRI) for evaluation of neurological disorders has increased in the past two decades. This has led to an increased detection of incidental findings on brain MRI. The most common of these asymptomatic abnormalities are white matter lesions that are interpreted as demyelinating based on radiological criteria. However, in the absence of associated clinical symptoms suggestive of multiple sclerosis (MS), a definite diagnosis of MS can't be made in patients with these incidental white matter lesions. These patients are diagnosed as CIS (clinically isolated syndrome) and RIS (radiologically isolated syndrome). Using the revised McDonald criteria now allows some patients who would have been diagnosed with $\mathrm{CIS}$ to be diagnosed as having MS before a second episode.

Method: Sixty one patients, 40 females and 21 males, age ranged between 15 years and 58 years, were included in our study. In addition to a detailed medical and neurological history and examination, CSF and blood analysis for oligoclonal bands and IgG index were performed for all patients.

Result: 41 patients had positive oligoclonal bands and IgG index. After clinical, MRI results and laboratory results $44(72.1 \%)$ were diagnosed CIS and 17 (27.9\%) were RIS.

Conclusion: Diagnosis of MS not depend only on MRI finding but need clinical and laboratory work up including CSF and blood analysis for oligoclonal bands and IgG index to confirm diagnosis.

\section{Introduction}

The easy and wide availability of brain MRI in the last decades has led to its increasing use in evaluation of a variety of neurological symptoms. Given its widespread use, it is common to detect some incidental findings in patients undergoing brain MRI for unrelated medical indications, such as head trauma or headache. The most common of these incidental abnormalities are white matter lesions that based on their appearance, location, and distribution are consistent with demyelination or MS (multiple sclerosis) but are not associated with any clinical symptoms suggesting MS [1]. Diagnosis of MS is based on demonstrating dissemination in space and time on MRI and excluding other neurological disorders that can clinically and radiologically mimic MS [2]. MRI criteria for MS are based on the presence of focal lesions in the white and /or gray matter of the CNS, which are considered typical for this condition in terms of distribution, morphology, evolution, and signal abnormalities on conventional MRI sequences (T2weighted, FLAIR, pre- and post-contrast T1-weighted scans) [3]. According to the 2017 revision of McDonald criteria, including two major changes, first is the early diagnosis of MS in patients with CIS with dissemination in space and +ve OCBs, without waiting for dissemination in time, second is, symptomatic or asymptomatic MRI lesions (except those in the optic nerve) can be considered as dissemination in space or time [4]. Incidental MR imaging findings resembling MS without typical MS symptoms are termed RIS, it was introduced in 2009 by Okuda to categorize incidental WM lesions 
suggestive of demyelinating disease in patients without typical MS symptoms and no better explanation for the MR imaging anomalies [5]. CSF analysis was one of the main paraclinical diagnostic criteria for MS (it is mandatory in the 2017 revision of McDonald criteria for MS); nowadays, it is of great importance in differential diagnosis of MS [4]. The presence of $\geq 2$ OCBs in the CSF have a positive predictive value of $97 \%$, negative predictive value of $84 \%$, sensitivity of $91 \%$, and specificity of $94 \%$ for developing (relapsing remitting MS) RRMS after a CIS [5] (Tables 1,2).

The condition to be termed CIS, the episode should last for at least 24 hour, and occur in the absence of fever or infection, with no clinical features of encephalopathy [7]. A retrospective review of brain MRI showed that 58\% had white matter T2 hyperintensities and that the prevalence of MRI findings meeting the Barkhof criteria for dissemination in space in a consecutive series of patients imaged for headaches ranged from $2.4 \%$ to $7.1 \%$ [8]. Several risk factors of conversion from RIS to MS have been discussed (Table 3 ).

\section{Aim of the study}

This study was prospective study that aimed to confirm the diagnosis of MS in patient presenting with neurological disorder and MRI brain finding suggestive MS.

\section{Patients and methods}

The study was carried out on sixty one patient aged $\geq 15$ years and less than 58 years of both sex attended the outpatient clinic or admitted at Neurology Department of Al-Jadaani and Bugshan Hospitals, Jeddah, KSA during the period from first January 2017 to the end of March 2018. Based on the digital radiological information system and digital patient charts, all patients undergoing a brain MRI and had radiological finding fulfilling the criteria of CIS or RIS during this period were included in the study. All MRI examinations were performed in the regular clinical setting in one of two $1.5 \mathrm{~T}$ MRI machines (gadolinium enhanced T1, T2, FLAIR and Diffusion sequences were done for all patients), MRI was interpreted by radiologist. This study had been approved by ethical committee of Al-Jadaani and Bugshan Hospitals. Patients who diagnosed MS,

Table 1: Proposed diagnostic criteria for radiologically isolated syndrome [5]

A- The presence of incidentally identified CNS white matter anomalies meeting the following MRI criteria

1. Ovoid, well-circumscribed and homogeneous foci with or without involvement of the corpus callosum

2. T2 hyperintensities measuring $>3 \mathrm{~mm}$ and fulfilling Barkhof criteria (at least three out of four; table 2) for dissemination in space

3. CNS white matter lesions not consistent with a vascular pattern

B- No historical accounts of remitting clinical symptoms consistent with neurological dysfunction

C- The MRI findings do not account for clinically apparent impairments in social, occupational or generalized areas of functioning

D- The MRI findings are not due to the direct physiological effects of substances (recreational drug abuse, toxic exposure) or a medical condition

E- Exclusion of individuals with MRI phenotypes suggestive of leukoaraiosis or extensive white matter pathology lacking involvement of the corpus callosum

F- The CNS MRI anomalies are not better accounted for by another disease process

Table 2: Barkhof's proposed MRI criteria for MS [6].

\begin{tabular}{|c|c|}
\hline T2 lesions & $\geq 9$ T2 hyperintense or $\geq 1$ gadolinium enhancing \\
\hline Infratentorial & lesions $\geq 1$ \\
\hline Juxtacortical & lesions $\geq 1$ \\
\hline Periventricular & lesions $\geq 4$ \\
\hline
\end{tabular}

Table 3: Clinical and radiological predictors which increase the risk of clinical progression in radiologically isolated syndrome [9].

Asymptomatic spinal cord lesions (especially cervical cord lesions)

Infratentorial lesions

A higher number of T2 lesions in MRI

Pathological visual evoked potential

Younger age

Oligoclonal bands and/or a pathological IgG index in combination with more than nine T2-lesions on the initial MR examination 
acute disseminated encephalomyelitis, autoimmune disease, cerebrovascular stroke, or patients who refused to participate in the study were excluded from the study. All patients included in the study were subjected to complete neurological history and examination. Detailed history of neurological symptoms (onset, duration, course and aggravating factors) and complete neurological examination including mental state, cranial nerves, motor, sensory systems and cerebellum were done for all patients. CSF and blood samples (in parallel) for oligoclonal bands and IgG index were done for all patients. Appropriate statistical methods were applied and the results were tabulated accordingly. $\mathrm{P}<0.05$ was considered significant.

\section{Results}

In this study, 61 patient were included, 40 females (65.6\%) and 21 males (34.4\%), the mean age of the patient were (31.11 \pm 10$)$. The mean age was higher among male (37.19 \pm 10.65$)$ than females $(27.92 \pm 8.08)$ with significant difference. Regarding the 44 patient with CIS, the most presenting symptom was acute optic neuritis, affecting 14 (31.8\%) patients, then 13 (29.5\%) patients with motor presentations, either hemiparesis $(11.5 \%)$ or paraparesis $(9.8 \%)$. Regarding patients with RIS, most presenting symptom was headache, affecting 7 (41.2\%) patients, as 4 patients with tension headache and 3 patients with migraine, then $3(17.6 \%)$ patients with loss of consciousness, (Table 4-6). The results of CSF and blood analysis for oligoclonal bands were 37 patients $(60.7 \%)$ had positive result and 24 patients $(39.3 \%)$ had negative results. After clinical, MRI finding and laboratory results 44 patients (72.3\%) were consistent with diagnosis of CIS (14 males and 30 females) and 17 patient $(27.7 \%)$ with RIS (7 males and 10 females).

\begin{tabular}{|c|c|c|c|c|}
\hline \multicolumn{1}{|l|}{ Table 4: Demographic data of studied patients. } \\
\hline Variable & CIS (N= 44) & RIS (N= 17) & Total (N= 61) & P value \\
\hline Sex & & & & \\
Male & $14(31.8 \%)$ & $7(41.2 \%)$ & $21(34.4 \%)$ & 0.216 \\
Female & $30(68.2 \%)$ & $10(58.8 \%)$ & $40(65.6 \%)$ & \\
\hline Age & & & & 0.454 \\
Male & $38.1 \pm 9.6$ & $35.8 \pm 12.6$ & $37.2 \pm 10.7$ & 0.639 \\
Female & $27.2 \pm 7.4$ & $30.1 \pm 9.9$ & $27.8 \pm 8.1$ & 0.332 \\
Total & $30.5 \pm 9.5$ & $32.6 \pm 11.2$ & $31.11 \pm 10$ & 0.001 \\
\hline P value & 0.001 & 0.302 & & \\
\hline
\end{tabular}

Table 5: Results of laboratory Oligoclonal band and IgG index.

\begin{tabular}{|c|c|c|c|c|c|c|}
\hline \multirow{2}{*}{ Table 5: Results of laboratory Oligoclonal band and IgG index. } \\
\hline \multirow{2}{*}{ Variable } & \multicolumn{2}{|c|}{ CIS $(\mathbf{n = 4 4 )}$} & \multicolumn{2}{|c|}{ RIS $(\mathbf{n = 1 7 )}$} & \multicolumn{2}{c|}{ Total $(\mathbf{n = 6 1 )}$} \\
\cline { 2 - 7 } & Number & Percent & Number & Percent & Number & Percent \\
\hline OCB & 33 & $(75 \%)$ & 4 & $(23.5 \%)$ & 37 & $(60.7 \%)$ \\
\hline IgG index & 31 & $(70.5 \%)$ & 4 & $(23.5 \%)$ & 35 & $(57.4 \%)$ \\
\hline
\end{tabular}

\begin{tabular}{|c|c|c|c|c|}
\hline Clinical presentation & Number & Percent & CIS $(N=44)$ & RIS ( $N=17)$ \\
\hline Optic neuritis & 14 & $22.9 \%$ & $14(31.8 \%)$ & 0 \\
\hline Weakness & 13 & $21.3 \%$ & $13(29.5 \%)$ & 0 \\
\hline Hemiparesis & 7 & $11.5 \%$ & 7 & 0 \\
\hline Paraparesis & 6 & $9.8 \%$ & 6 & 0 \\
\hline Brain stem & 8 & $13.11 \%$ & $5(11.4 \%)$ & $3(17.6 \%)$ \\
\hline Vertigo & 3 & $4.9 \%$ & 1 & 2 \\
\hline Ocular nerve & 2 & $3.3 \%$ & 2 & 0 \\
\hline INO & 2 & $3.3 \%$ & 2 & 0 \\
\hline Bell's palsy & 1 & $1.6 \%$ & 0 & 1 \\
\hline Headache & 7 & $11.5 \%$ & 0 & $7(41.3 \%)$ \\
\hline $\mathrm{TH}$ & 4 & $6.6 \%$ & 0 & 4 \\
\hline Migraine & 3 & $4.9 \%$ & 0 & 3 \\
\hline Sensory symptoms & 6 & $9.8 \%$ & $4(9 \%)$ & $2(11.8 \%)$ \\
\hline TN & 5 & $8.2 \%$ & 3 & 2 \\
\hline Hemihyposthesia & 1 & $1.6 \%$ & 1 & 0 \\
\hline Ataxia & 7 & $11.5 \%$ & $7(15.9 \%)$ & 0 \\
\hline Recurrent LOC & 3 & $4.9 \%$ & 0 & $3(17.7 \%)$ \\
\hline Dizziness & 3 & $4.9 \%$ & $1(2.3 \%)$ & $2(11.8 \%)$ \\
\hline
\end{tabular}




\section{Discussion}

The diagnosis of MS is primary clinical and is dependent on the demonstration of neurologic signs and symptoms subsequent to white matter lesions on MRI. It depends largely on the results of MRI examination. Incidental MRI findings suggestive of MS without typical MS symptoms defined as RIS [10].

The association of RIS and MS is also strengthened in that both patient groups show similarities in both qualitative and quantitative MRI measurements $[11,12]$. Patients should not be diagnosed with MS on the basis of MRI findings alone, and at least one clinical event consistent with acute demyelination should remain a cornerstone for diagnosis [13]. Our study was included 61 patients, 40 females and 21 males. After clinical, MRI finding and laboratory results, 44 patients were diagnosed as CIS and 17 patient were diagnosed as RIS. Male to female ratio was 1:1.9 among studied patients and 1:2.14 among patients diagnosed CIS. These results were agree with $[14,15]$ they reported that MS is more common in women than in men, and has increased over the last decades from a female-to-male ratio of 1.4 in 1955 to 2.3 in 2000 . This corresponds to a lifetime risk of $2.5 \%$ in women compared to $1.4 \%$ in men. The mean age of patients was 31ys and for patients with CIS 30.5. It was higher among male than female with significant difference especially among patients with CIS that consistent with [16] he reported that the incidence of MS is low in childhood and increases after the age of 18, reaching a peak between 20 and 40 years (mean age of 30 years) with women being affected approximately 2-5 years earlier than men [17].

Reported that, the onset of MS in 85\% of young adults (aged 20-45 years). Regarding results of OCB, $75 \%$ of patients with CIS have positive OCB in CSF while in RIS only $23 \%$ has positive results. These results was partially agreed with [18-25] they mentioned that, Sixty to seventy percent of patients with CIS have positive OCBs and with [26] he reported that the positive OCB were detected in $61 \%$ of patients with CIS [27]. Found CSF oligoclonal banding having sensitivity between 69-91\% for diagnosis of MS. Regarding the clinical presentation, optic neuritis was most common presentation for CIS patients (31\%) and headache was most common presentation for RIS patients (41\%) [28]. Reported that, acute demyelinating optic neuritis is the presenting symptom in about $20 \%$ of MS patients and affects about half of MS patients at some point in the disease course and also [16] reported that, typical clinical presentations of RRMS are optic neuritis (in about $20 \%$ of cases this is the initial symptom). Headache is the most common reason for performing the initial MRI unveiling RIS, but it is unclear if there is a causative relationship between the incidental MRI findings and the headaches [29]. A study by Liu et al., showed that among patients undergoing MRI of the brain due to headaches, MRI findings fulfilling the Barkhof criteria are common [8].

\section{Conclusion}

The diagnosis of MS not depend only on MRI finding but need clinical presentation consistent with diagnosis of MS and CSF analysis for oligoclonal bands to confirm diagnosis.

\section{References}

1. Leahy H, Garg N. Radiologically Isolated Syndrome: An Overview Neurol. 2013; 5: 22-26. Ref.: https://tinyurl.com/ycly3ta7

2. Polman $\mathrm{CH}$, Reingold SC, Banwell $\mathrm{B}$, Clanet $\mathrm{M}$, Cohen JA, et al. Diagnostic criteria for multiple sclerosis: 2010 revisions to the McDonald criteria. Ann Neurol. 2011; 69: 292-302. Ref.: https://tinyurl.com/y9vt4n2h

3. Chard DT, Dalton CM, Swanton J, Fisniku LK, Miszkiel KA, et al. MRI only conversion to multiple sclerosis following a clinically isolated syndrome. J Neurol Neurosurg Psychiatry. 2011; 82: 176-179. Ref.: https://tinyurl.com/ydh8p929

4. Thompson AJ, Banwell BL, Barkhof F, Carroll WM, Coetzee T, et al. Diagnosis of multiple sclerosis: 2017 revisions of the McDonald criteria. Lancet Neurol. 2018; 17: 162-173. Ref.: https://tinyurl.com/yblx28l8 
5. Masjuan J, Alvarez-Cermeno JC, Garcia-Barragan N, Díaz-Sánchez M, Espiño M, et al. Clinically isolated syndromes: a new oligoclonal band test accurately predicts conversion to MS. Neurology. 2006; 66: 576-578. Ref.: https://tinyurl.com/y7hjfude

6. Sellner J, Schirmer L, Hemmer B, Mühlau M. The radiologically isolated syndrome: take action when the unexpected is uncovered. J Neurol. 2010; 257: 1602-1611. Ref.: https://tinyurl.com/ydy3sydo

7. Miller DH, Chard DT, Ciccarelli O. Clinically isolated syndromes. Lancet Neurol. 2012; 11: 157-169. Ref.: https://tinyurl.com/yb967rg7

8. Liu S, Kullnat J, Bourdette D, Simon J, Kraemer DF, et al. Prevalence of brain magnetic resonance imaging meeting Barkhof and McDonald criteria for dissemination in space among headache patients. Mult Scler. 2013; 19: 1101-1115. Ref.: https://tinyurl.com/y6wubu7v

9. Polman $\mathrm{CH}$, Reingold SC, Edan G, Filippi M, Hartung HP, et al. Diagnostic criteria for multiple sclerosis: 2005 revisions to the "McDonald Criteria". Ann Neurol. 2005; 58: 840-846. Ref.: https://tinyurl.com/y7tfpuzn

10. Okuda DT, Mowry EM, Beheshtian A, Waubant E, Baranzini SE, et al. Incidental MRI anomalies suggestive of multiple sclerosis: the radiologically isolated syndrome. Neurology. 2009; 72: 800-805. Ref.: https://tinyurl.com/y95weesd

11. Amato MP, Hakiki B, Goretti B, Rossi F, Stromillo ML, et al . Association of MRI metrics and cognitive impairment in radiologically isolated syndromes. Neurology. 2012; 78: 309-314. Ref.: https://tinyurl.com/ydesg9bo

12. Stromillo ML, Giorgio A, Rossi F, Battaglini M, Hakiki B, et al. Brain metabolic changes suggestive of axonal damage in radiologically isolated syndrome. Neurology. 2013; 80: 2090-2094. Ref.: https://tinyurl.com/yavdsygg

13. Miller DH, Chard DT, Ciccarelli O. Clinically isolated syndromes. Lancet Neurol. 2012; 11: 157-169. Ref.: https://tinyurl.com/yb967rg7

14. Alonso A, Hernán MA. Temporal trends in the incidence of multiple sclerosis: a systematic review (review). Neurology. 2008; 71: 129-135. Ref.: https://tinyurl.com/yd34lrfw

15. Koch-Henriksen N, Sørensen PS. The changing demographic pattern of multiple sclerosis epidemiology (review). Lancet Neurol. 2010; 9: 520-532. Ref.: https://tinyurl.com/ya3n57el

16. Confavreux C, Vukusic S. Natural history of multiple sclerosis: a unifying concept. Brain. 2006; 129 : 606-616. Ref.: https://tinyurl.com/yd2ksepd

17. Miller D, Barkhof $F$, Montalban X, Thompson A, Filippi M. Clinically isolated syndromes suggestive of multiple sclerosis, part II: non-conventional MRI, recovery processes, and management. Lancet Neurol. 2005; 4: 341-348. Ref.: https://tinyurl.com/ybo6ty75

18. Miller D, Barkhof F, Montalban X, Thompson A, Filippi M. Clinically isolated syndromes suggestive of multiple sclerosis, part I: natural history, pathogenesis, diagnosis, and prognosis. Lancet Neurol. 2005; 4: 281-288. Ref.: https://tinyurl.com/ycsj39mm

19. Awad A, Hemmer B, Hartung HP, Kieseier B, Bennett JL, et al. Analyses of cerebrospinal fluid in the diagnosis and monitoring of multiple sclerosis. J Neuroimmunol. 2010; 219: 1-7. Ref.: https://tinyurl.com/yd7eq7d3

20. Zipoli V, Hakiki B, Portaccio E, Lolli F, Siracusa G, et al. The contribution of cerebrospinal fluid oligoclonal bands to the early diagnosis of multiple sclerosis. Mult Scler. 2009; 15: 472-478. Ref.: https://tinyurl.com/y93ctf87

21. Paolino E, Fainardi E, Ruppi P, Tola MR, Govoni V, et al. A prospective study on the predictive value of CSF oligoclonal bands and MRI in acute isolated neurological syndromes for subsequent progression to multiple sclerosis. J Neurol Neurosurg Psychiatry. 1996; 60: 572-575. Ref.: https://tinyurl.com/y99ot95u

22. Sastre-Garriga J, Tintore M, Rovira A, Grivé E, Pericot I, et al. Conversion to multiple sclerosis after a clinically isolated syndrome of the brainstem: cranial magnetic resonance imaging, cerebrospinal fluid and neurophysiological findings. Mult Scler. 2003; 9: 39-43. Ref.: https://tinyurl.com/y9poolcx

23. Tintore M, Rovira A, Brieva L, Grivé E, Jardí R, et al. Isolated demyelinating syndromes: comparison of CSF oligoclonal bands and different MR imaging criteria to predict conversion to CDMS. Mult Scler. 2001; 7: 359-363. Ref.: https://tinyurl.com/y9weq69j

24. Avasarala JR, Cross AH, Trotter JL. Oligoclonal band number as a marker for prognosis in multiple sclerosis. Arch Neurol. 2001; 58: 2044-2045. Ref.: https://tinyurl.com/ycy9nq84 
25. Sellner J, Schirmer L, Hemmer B, Mühlau M. The radiologically isolated syndrome: take action when the unexpected is uncovered? J Neurol. 2010; 257: 1602-1611. Ref.: https://tinyurl.com/ydy3sydo

26. Schwenkenbecher $P$, Sarikidi A, Bönig L, Wurster U, Bronzlik $P$, et al. Clinically Isolated Syndrome According to McDonald 2010: Intrathecal IgG Synthesis Still Predictive for Conversion to Multiple Sclerosis. Int J Mol Sci. 2017; 18: 2061. Ref.: https://tinyurl.com/yc4cd9dk

27. Schaffler N, Kopke S, Winkler L, Schippling S, Inglese M, et al. Accuracy of diagnostic tests in multiple sclerosis--a systematic review. Acta Neurol Scand. 2011;124: 151-164. Ref.: https://tinyurl.com/y8fekjsb

28. Balcer LJ. Clinical practice. Optic neuritis. N Engl J Med. 2006; 354: 1273-1280. Ref.: https://tinyurl.com/y92orlma

29. Granberg T, Martola J, Kristoffersen-Wiberg M, Aspelin P, Fredrikson S. Radiologically isolated syndrome-incidental magnetic resonance imaging findings suggestive of multiple sclerosis, a systematic review. Mult Scler. 2013; 19: 271-280. Ref.: https://tinyurl.com/ybgwowma 